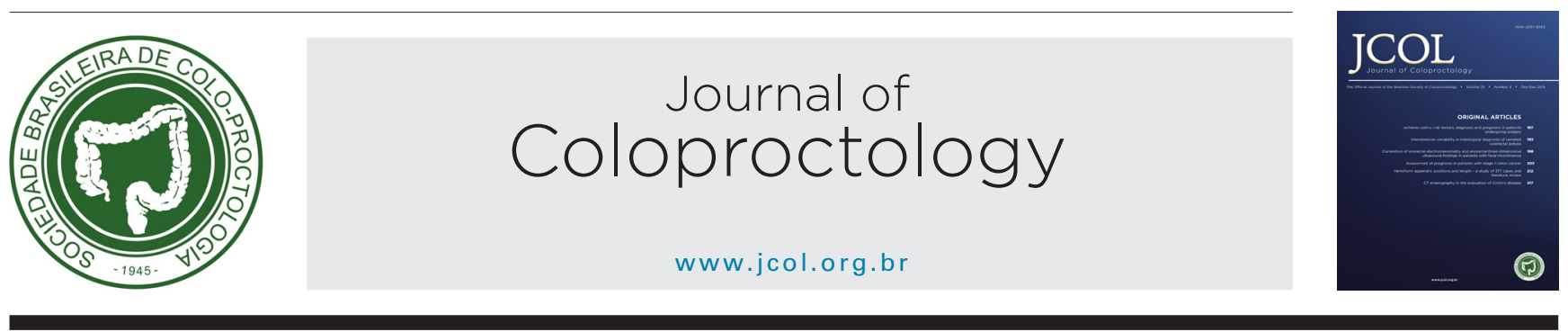

\title{
Editorial
}

\section{Confocal laser endomicroscopy in inflammatory bowel diseases: future, reality or dream?}

\section{Introduction}

Confocal endomicroscopy (CLE) is a new endoscopic technique that allows to obtain high-resolution layer lining of the gastrointestinal tract imaging. This technique is based on illumination of the mucosa with a laser. In this process, the laser is absorbed by a fluorescent agent and the reflected light is captured. The laser is focused at a selected depth in the tissue of interest and the reflected light is then redirected to a detection system for the same lens, excluding the reflected light from other parts of the mucosa. ${ }^{1-3}$ This increases significantly the spatial resolution of the images obtained, and, if the capturing is very magnified, high-resolution images are produced. This technique can be applied during the examination of endoscopy, and cellular and subcellular structures, with extreme detail, as in the conventional microscope. ${ }^{1-3}$

Currently pCLE systems is a probe-based system (Mauna Kea Technologies, Paris, France Cellvizio); the probe is placed through the biopsy channel of a conventional endoscope. The depth of view is up to $250 \mathrm{~mm}$ with the endoscopic system and about $120 \mathrm{~mm}$ with the probe-based system. A limited area can be examined; no more than $700 \mathrm{~mm}$ in the endoscopic-based system and less with the probe-based system. CLE systems are optimized using a contrast agent. The most widely used agent is intravenous fluorescein, which is FDA-approved for ophthalmologic imaging of blood vessels when used with a laser scanning ophthalmoscope. ${ }^{1-4}$

The use of confocal laser endomicroscopy (CLE), also known as confocal fluorescent endomicroscopy and optical endomicroscopy, allows in vivo microscopic imaging of cells during endoscopy. CLE is proposed for a variety of purposes, especially as a real-time alternative to histology during colonoscopy and for targeting areas to undergo biopsy in patients with inflammatory bowel disease (IBD), differentiation between neoplastic and benign polyps..$^{2,3}$

One of the concerns of all the professionals who follow up the patients with longstanding IBD is the emergence of cancer in this specific group. It is estimated that the possibility of onset of colorectal cancer is 2-5 times higher in this group than in the general population of the same age. ${ }^{4}$

The CLE technique offers some disadvantages that must be considered. Among then, we can enumerate:

(1) The learning curve is relatively long and depends on the training of the specialist to obtain good performance and interpretation of $\mathrm{CLE}^{5-8}$;

(2) The high cost of probe to be used. The recommendation is to change the probe after 20 exams. Also the cost of the equipment to read the images ${ }^{5,6}$;

(3) The operator dependency with regard the difficult in maintaining the stability of the probe, for interpretation of morphologic features, and limited depth of penetration of the tool. ${ }^{9}$

(4) The impossibility of examining the entire surface of the colon using only the endomicroscopic mode. A combination of endomicroscopy with chromoendoscopy in patients with ulcerative colitis is necessary. Chromoendoscopy unmasks circumscript lesions and confocal laser endomicroscopy can be used to predict intraepithelial neoplasias with high accuracy. ${ }^{1-3}$

We also enumerate some advantages and indications:

(1) pCLE with fluorescein sodium allowed us to analyze the cellular structure, connective tissue, and blood cells of the colonic mucosa in vivo. ${ }^{1-3,5}$

(2) pCLE could promote in vivo virtual histology and may leading to an optimized rapid diagnosis of colonic lesions that has important implications for clinical therapy (e.g., endoscopic resection of neoplastic tissue). ${ }^{6-8}$

(3) pCLE allows in vivo microscopic analysis with impressive imaging of living cells during colonoscopy and, in some cases, could establish after identifying the lesion, defining the security areas after remove the lesion. 1,5,6,9 
(4) pCLE promotes the possibility of diagnostic in vivo. This helps significantly on reduction of the number of biopsies to be performed, restricting to target biopsy and so limiting the adverse reactions that could occur during multiple randomized biopsies..$^{1,5}$

(5) pCLE enables to distinguish, with high accuracy, the differentiation between hyperplastic polyps from adenomatous polyps and gives support for immediate resection of suspicious lesions. The differentiation is usually difficult when we use only the macroscopic criteria. ${ }^{5,6}$

\section{Discussion}

Currently pCLE has emerged as a new tool of endoscopic imaging modality enabling real-time in vivo histology of the cellular mucosal layer at a magnification of 1000 -fold. It is necessary application of an exogenous fluorescence agent. These agents can either be applied systemically (fluorescein) or topically (acriflavine hydrochloride, cresyl violet) to the intestinal mucosa. The use of fluorescence agents enables imaging of mucosal structures and emphasizes the imaging of features such as the capillary, architectural, and cellular patterns.

Inflammatory bowel disease is an excellent example of the application of pCLE in clinical practice.

Patients with longstanding UC are at increased risk to development neoplasia, but it is difficult to detect by white light endoscopy and random biopsies is necessary to identified abnormalities in the mucosa. On the other hand, panendomicroscopy of the entire colon is not possible. An option has been combined pCLE with chromoendoscopy for optimized and increases the findings and care of patients under surveillance for longstanding ulcerative colitis. In this setting, chromoendoscopy is used to increase a possibility to discover suspicious regions and PCLE is used for their immediate characterization. An important finding was a possibility to predict of normal mucosa with an accuracy of over $99 \%$ in optical biopsies (with conventional histopathology as the gold standard).

Although until now there is not a close correspondence between the conventional endoscopic images and histological assessment, this association of tools remains the best way to diagnose accurately and then treat as early as possible many diseases, especially of the colorectal tract, including chronic inflammatory, preneoplastic, and neoplastic diseases. Based on the finding that conventional colonoscopy is not always able to differentiate between neoplastic and nonneoplastic lesions, in recent years, several studies highlighted the potential use of CLE, as a new emerging technique, in the screening of patients for early colorectal cancer detection and prevention. , $6,9^{-1}$

A prospective study was conducted involved 42 patients were undergoing a colonoscopy and confocal laser endoscopy. Acriflavine hydrochloride and fluorescein sodium were used both yielded high-quality images. Whereas acriflavine hydrochloride be strongly labeled to the superficial epithelial cells, fluorescein sodium offers deeper imaging into the lamina propria. Subsurface analysis during confocal laser endoscopy allowed detailed analysis of cellular structures. The presence of neoplastic changes could be predicted with high accuracy (sensitivity, 97.4\%; specificity, 99.4\%; accuracy, $99.2 \%)^{1}$

Multicenter study was performed for assessing fluorescein assisted pCLE. No major adverse events occurred in 2272 procedures, and mild adverse events were recorded in $1.4 \%$ of the procedures, including nausea, vomiting, transient hypotension, injection site erythema, rash, and epigastric pain. ${ }^{3}$

There are some alternatives of use which are primarily intended to improve the sensitivity of colonoscopy, as a chromoendoscopy. However, CLE is unique in sense that it is designed to immediately characterize the cellular structure of lesions. ${ }^{4}$ CLE can thus potentially be used to make a diagnostic of polyp histology, particularly in association with screening or surveillance colonoscopy, which could allow to leave in place the small hyperplastic lesions rather than remove them and send for histologic evaluation. This could reduce risks associated with biopsy and reduce the number of biopsies and histologic evaluations and certainly will improve the detection of dysplasia. This is an alternative to conducting random biopsies during surveillance. ${ }^{4,5,9}$

Recently, the use of CLE was reported in the study of colonic mucosa in patients with inflammatory bowel diseases and in particular in patients affected by ulcerative colitis. CLE has an important role in staging the patients with inflammatory bowel diseases. It can be used to assess the grading of colitis and to detect microscopic colitis in endoscopically silent segments. Moreover, CLE can be used in surveillance programs especially in high risk patients. Finally, CLE has been effectively used in diagnosing a biliary dysplasia/neoplasia in patients with primary sclerosing cholangitis, a pathological condition frequently associated with IBD, with a coexisting bile duct stricture..$^{5,6,9}$

Just like any other advanced and new endoscopy, CLE technique requires trainings and handling of the endoscopy and time to learn and interpret with confidence the images generated. ${ }^{6}$

The American Society of Gastrointestinal Endoscopy (ASGE) recommends that the endoscopies exams achieve $\geq 90 \%$ negative predictive value (NPV) and concordance of $\geq 90 \%$ saying that the NPV has to exceed $90 \%$ and the prediction of the surveillance interval should be correct in more than $90 \%$ of the cases. These data should always be achieved. ${ }^{5}$

CLE should be used to enhance the diagnostic arsenal in the evaluation of these indications, by providing microscopic information which improves the diagnostic performance of the physician. In order to actually implement this technology in the clinical routine, and to ensure good practice, the initial standardization and the continuing institutional training programs should be established. ${ }^{5-9}$

More studies give a very promising glance at possible future applications, however is need a larger prospective, multicenter trials to prove the advantage of this technique in daily practice. ${ }^{10,11}$

\section{Conclusion}

Confocal laser endomicroscopy (CLE) is a newly option that could give us an envision to investigate and to diagnostic the diseases related to gastrointestinal systems. It allows virtual 
histology of the mucosal layer in real time ongoing endoscopy. The images obtained through this new technology represent the beginning of a new era, that will permit detailed visualization of the living cells and the cellular structures that are below of the surface of the gut. 1,2,5,6,8,11,12

We will need more time to implement and make investments in this ultimate endoscopic imaging tool however, this is not a dream or future is now a reality.

\section{Funding}

Manoel Álvaro de F. Lins Neto is supported by the National Council for Scientific and Technological Development (CNPq), Brazil. Eliana Silva de Almeida is supported by the National Council for Scientific and Technological Development (CNPq), Brazil.

\section{Conflicts of interest}

The authors declare no conflicts of interest.

\section{REFERENCES}

1. Kiesslich R, Burg J, Vieth M, Gnaendiger J, Enders M, Delaney $\mathrm{P}$, et al. Confocal laser endoscopy for diagnosing intraepithelial neoplasias and colorectal cancer in vivo. Gastroenterology. 2004;127:706-13.

2. Goetz M, Kiesslich R. Advances of endomicroscopy for gastrointestinal physiology and diseases. Am J Physiol Gastrointest Liver Physiol. 2010;298:G797-806.

3. Wallace MB, Meining A, Canto MI, Fockens P, Miehlke S, Roesch $\mathrm{T}$, et al. The safety of intravenous fluorescein for confocal laser endomicroscopy in the gastrointestinal tract. Aliment Pharmacol Ther. 2010;31:548-52.

4. Iacucci M, Uraoka T, Fort Gasia M, Yahagi N. Novel diagnostic and therapeutic techniques for surveillance of dysplasia in patients with inflammatory bowel disease. Can J

Gastroenterol Hepatol. 2014;28:361-70.
5. McGill KS, Soetikno R, Rastogi A, Rouse RS, Sato T, Bansal A, et al. Endoscopists can sustain high performance for the optical diagnosis of colorectal polyps following standardized and continued training. Endoscopy. 2015;47:200-6.

6. Mascolo M, Staibano S, Ilardi G, Siano M, Vecchione ML, Esposito D, et al. Probe-based confocal laser endomicroscopy evaluation of colon preneoplastic lesions, with particular attention to the aberrant crypt foci, and comparative assessment with histological features obtained by conventional endoscopy gastroenterology. Res Pract. 2012;2012:1-6.

7. Kiesslich R. Optical diagnosis of colorectal polyps: can this be learned by everyone? Endoscopy. 2015;47:188-9.

8. Goetz M. Confocal laser endomicroscopy: applications in clinical and translational science-a comprehensive review. Int Sch Res Netw ISRN Pathol. 2012;2012:387145.

9. Wang KK, Carr-Locke LK, Singh SK, Neumann H, Bertani H, Galmiche JP, et al. Use of probe-based confocal laser endomicroscopy (pCLE) in gastrointestinal applications. A consensus report based on clinical evidence. United Eur Gastroenterol J. 2015;3:230-54.

10. Salvatori F, Siciliano S, Maione F, Esposito D, Masone S, Persico M, et al. Confocal laser endomicroscopy in the study of colonic mucosa in IBD patients: a review. Gastroenterol Res Pract. 2012;2012:525098.

11. Bisschops R. Confocal laser endomicroscopy: finally ready to change clinical practice? Gastrointest Endosc. 2011;74:781-3.

12. Li WB, Zuo XL, Li CQ, Zuo F, Gu XM, Yu T, et al. Diagnostic value of confocal laser endomicroscopy for gastric superficial cancerous lesions. Gut. 2011;60:299-306.

Manoel Alvaro Lins Neto ${ }^{a, b, *}$, Eliana Silva de Almeida ${ }^{a}$

a Universidade Federal de Alagoas (UFAL), Maceió, AL, Brazil

${ }^{\mathrm{b}}$ Service of Coloproctology, Hospital Universitário Prof. Alberto Antunes, Universidade Federal de Alagoas (UFAL), Maceió, AL, Brazil

*Corresponding author. E-mail: mlinsneto@gmail.com (M.A.L. Neto).

http://dx.doi.org/10.1016/j.jcol.2015.12.006

2237-9363/@ 2016 Published by Elsevier Editora Ltda. on behalf of Sociedade Brasileira de Coloproctologia. 